Pacific Journal of Mathematics

SOME ZERO SUM TWO-PERSON GAMES WITH MOVES IN 


\section{SOME ZERO SUM TWO-PERSON GAMES WITH MOVES IN THE UNIT INTERVAL}

\section{MARTIN Fox}

Introduction. Consider the following zero sum two person game. The players alternately choose points $t_{i} \in[0,1]$ for $i=1,2, \cdots, n$, the choice being made by player I if $i$ is odd and by player II if $i$ is even. After the $i$ th move the player who is to make the $(i+1)$ st move observes the value of $\phi_{i}\left(t_{1}, t_{2}, \cdots, t_{i}\right)$ where $\phi_{i}$ is some function on the $i$ dimensional closed unit cube to some set $A_{i}$. The payoff is $f\left(t_{1}, t_{2}, \cdots, t_{n}\right)$ where $f$ is a continuous, real-valued function.

If all the $\phi_{i}$ are constant we have the case of no information. Ville [1] showed that in this case such a game has a value. At the other extreme, if the $\phi_{i}$ are all one-to-one we have the case of perfect information so the game has a value.

The purpose of the present paper is to show that, in general, games of the form introduced in the first paragraph do not have values and to consider two cases in which they do. The counter-examples to be presented will be compared with Ville's classical example of a game on the unit square which has no value.

It is shown in $\S 2$ that the games considered always have values when $n=2$.

An example of a game with no value is presented in $\S 3$. In this example $n=3$ and the $\phi_{i}$ take only a finite number of values.

In $\S 4$ it is shown that the additional hypothesis of continuity of the $\phi_{i}$ is not sufficient to guarantee existence of a value. In that example $n=4$. The case $n=3$ with continuous $\phi_{i}$ remains unsolved.

Section 5 deals with a special case for which $n$ is arbitrary and yet the game has a value. In this case the $\phi_{i}$ each take only a finite number of values and each is constant on sets which are finite unions of $i$-dimensional generalized intervals.

1. Preliminary remarks. In this section the notation to be used in this paper will be introduced. This will be facilitated by the introduction of the normal forms of the games under consideration.

A pure strategy for player $\mathrm{I}$ is a vector $x=\left(x_{1}, x_{2}, \cdots, x_{[(n+1) / 2]}\right)$ where $x_{1} \in[0,1]$ and the $x_{i}$ for $i=2,3, \cdots,[(n+1) / 2]$ are functions on $A_{2 i-2}$ to $[0,1]$. If moves $t_{1}, t_{2}, \cdots, t_{2 i-2}$ have been made, then the $i$ th move made by player I (the $(2 i-1)$ st move in the game) will be $x_{i}\left(\phi_{2 i-2}\left(t_{1}, t_{2}, \cdots, t_{2 i-2}\right)\right)$. His first move will be $x_{1}$.

Received November 16, 1959. Excerpt from dissertation for the degree of Doctor of Philosophy, University of California, 1959. Partial support was given by Office of Ordnance Research, U.S. Army under Contract DA-04-200-ORD-171, Task Order 3. 
A pure strategy for player II is a vector $y=\left(y_{1}, y_{2}, \cdots, y_{[n / 2]}\right)$ where each $y_{i}$ is a function on $A_{2 i-1}$ to $[0,1]$. If moves $t_{1}, t_{2}, \cdots, t_{2 i-1}$ have been made, then the $i$ th move made by player II (the (2i)th move in the game) will be $y_{i}\left(\phi_{2 i-1}\left(t_{1}, t_{2}, \cdots, t_{2 i-1}\right)\right)$.

When player I uses the pure strategy $x$ and player II uses the pure strategy $y$ let $t_{i}(x, y)$ be the $i$ th move made in the game. The $t_{i}$ are defined recursively as follows:

$$
\begin{aligned}
t_{1}(x, y) & =x_{1} ; \\
t_{2 i}(x, y) & =y_{i}\left(\phi_{2 i-1}\left(t_{1}(x, y), t_{2}(x, y), \cdots, t_{2 i-1}(x, y)\right)\right) \\
& \text { for } i=1,2, \cdots,[n / 2] ; \\
t_{2 i-1}(x, y) & =x_{i}\left(\phi_{2 i-2}\left(t_{1}(x, y), t_{2}(x, y), \cdots, t_{2 i-2}(x, y)\right)\right) \\
& \text { for } i=2,3, \cdots,[(n+1) / 2] .
\end{aligned}
$$

The payoff function is given by $M(x, y)=f\left(t_{1}(x, y), t_{2}(x, y), \cdots, t_{n}(x, y)\right)$. The payoff as a function of mixed strategies will also be denoted by $M$.

In our case, since the moves are points in $[0,1]$, the strategy spaces $X$ and $Y$ are products, usually infinite dimensional, each coordinate space being $[0,1]$. Hence, the choice of a strategy by player $I$ is equivalent to the choice of a distribution function $F$ on $X$. It will be convenient to let the space $P$ of mixed strategies for player I be the family of all distribution functions on $X$ which assign probability 1 to a finite subset of $X$. The same will be done for $Q$, the space of mixed strategies for player II.

If $H$ is a distribution function on the real line and $S$ is any subset of the real line which is Borel measurable, we will let $H S$ be the probability assigned to $S$ by $H$.

For $F \in P$ we let $F_{i, \alpha}$ denote the marginal distribution function of the coordinate of player I's strategy which corresponds to his $i$ th move when $\phi_{2 i-2}=\alpha$. Similar notation will be used for $G \in Q$.

2. The case $n=2$. In this section it will be shown that any game $\mathscr{G}$ of the type given in the introduction for which $n=2$ has a value. It is not even necessary to assume that $\phi_{1}$ is a measurable function.

For any $\alpha \in A_{1}$ let $\mathscr{G}(\alpha)=\left(\phi_{1}^{-1}(\alpha),[0,1], M_{\alpha}\right)$ where $M_{\alpha}$ is $f$ restricted to $\phi_{1}^{-1}(\alpha) \times[0,1]$. It follows by the proof used for Ville's minimax theorem that each $\mathscr{G}(\alpha)$ has a value $v(\alpha)$. Let

$$
v=\sup _{\alpha \in A_{1}} v(\alpha) .
$$

Fix $\varepsilon>0$ and let $\alpha^{*}$ be such that $v\left(\alpha^{*}\right)>v-\varepsilon$. For each $\alpha \in A_{1}$ let $F^{(\alpha)}$ and $G^{(\alpha)}$ be $\varepsilon$-good strategies for players I and II, respectively, in $\mathscr{G}(\alpha)$. The distribution function $F^{(\alpha)}$ assigns probability 1 to a finite subset of $\phi_{1}^{-1}(\alpha)$. Since $F^{\left(\alpha^{*}\right)}$ is a distribution function on $[0,1]$ which 
is the strategy space for player I in $\mathscr{G}$, it can also be used as a strategy in $\mathscr{G}$. Let $y$ be any pure strategy for player II in $\mathscr{G}$. Since $y_{1}\left(\alpha^{*}\right) \in[0,1]$, it follows that $y_{1}\left(\alpha^{*}\right)$ is a pure strategy for player II in $\mathscr{G}\left(\alpha^{*}\right)$. Hence,

$$
\begin{aligned}
M\left(F^{\left(\alpha^{*}\right)}, y\right) & =\int_{\phi_{1}^{-1}\left(\alpha^{*}\right)} f\left(t, y_{1}\left(\alpha^{*}\right)\right) F^{\left(\alpha^{*}\right)}(d t) \\
& =\int M_{\alpha^{*}}\left(t, y_{1}\left(\alpha^{*}\right)\right) F^{\left(\alpha^{*}\right)}(d t) \\
& =M_{\alpha^{*}}\left(F^{\left(\alpha^{*}\right)}, y_{1}\left(\alpha^{*}\right)\right) \\
& >v\left(\alpha^{*}\right)-\varepsilon>v-2 \varepsilon
\end{aligned}
$$

Let $G$ be any strategy for player II in $\mathscr{G}$ such that $G_{1, \alpha}=G^{(\alpha)}$ for all $\alpha \in A_{1}$. Let $x$ be any pure strategy for player I in $\mathscr{C}$. For some $\alpha \in A_{1}$ it must be true that $x \in \phi_{1}^{-1}(\alpha)$ so that $x$ is also a pure strategy for player I in $\mathscr{C}(\alpha)$. Then,

$$
\begin{aligned}
M(x, G) & =\int f(x, t) G_{1, \alpha}(d t) \\
& =\int M_{\alpha}(x, t) G^{(\alpha)}(d t) \\
& =M_{\alpha}\left(x, G^{(\alpha)}\right) \\
& <v(\alpha)+\varepsilon \leqq v+\varepsilon .
\end{aligned}
$$

From the two inequalities obtained above it follows that the value of $\mathscr{G}$ is $v$.

3. A counter-example for $n=3$. In this section the counterexample for $n=3$ will be given. The functions $\phi_{i}(i=1,2)$ each take only a finite number of values. The similarity of this example to Ville's example will be discussed.

For this example let

$$
\begin{aligned}
\phi_{1}\left(t_{1}\right) & \equiv 0 \\
\phi_{2}\left(t_{1}, t_{2}\right) & =\left\{\begin{array}{l}
-1 \text { if } t_{1}=0 \text { or } 0<\min \left(t_{2}, 1-t_{2}\right) \leqq t_{1} \\
t_{2} \text { if } t_{2}=0 \text { or } 1 \text { and } t_{1} \neq 0 ; \\
2 \text { if } 0<t_{1}<t_{2} \leqq \frac{1}{2} \\
3 \text { if } 0<t_{1}<1-t_{2}<\frac{1}{2}
\end{array}\right. \\
f\left(t_{1}, t_{2}, t_{3}\right) & =-\left|t_{3}-t_{2}\right| .
\end{aligned}
$$

Let $F$ be any strategy for player I. Fix $\varepsilon>0$ and let $\delta \in(0, \varepsilon)$ be sufficiently small so that $F_{1}(0, \delta)<\varepsilon$. Let $G\{\delta\}=G\{1-\delta\}=1 / 2$. Then, 


$$
\begin{aligned}
M(F, G) \leqq & -\frac{1}{2}\left(F_{1}[\delta, 1]+F_{1}\{0\}\right)\left[\int\left|t_{3}-\delta\right| F_{2,-1}\left(d t_{3}\right)\right. \\
& \left.+\int\left|t_{3}-(1-\delta)\right| F_{2,-1}\left(d t_{3}\right)\right] \\
< & -\frac{1}{2}(1-\varepsilon)\left[\left(\frac{1}{2}-\delta\right)+\left(1-\delta-\frac{1}{2}\right)\right]<-\frac{1}{2}+\frac{3}{2} \varepsilon
\end{aligned}
$$

so that

$$
\sup _{F} \inf _{G} M(F, G) \leqq-\frac{1}{2}
$$

Let $G$ be any strategy for player II. Fix $\varepsilon>0$ and let $x_{1} \in(0,1 / 2)$ be sufficiently small so that $G\left(0, x_{1}\right]+G\left[1-x_{1}, 1\right)<\varepsilon$.

Let

$$
x_{2}(\alpha)=\left\{\begin{array}{l}
\frac{1}{2} \text { if } \alpha=-1 ; \\
\alpha \text { if } \alpha=0 \text { or } 1 ; \\
\frac{1}{4} \text { if } \alpha=2 ; \\
\frac{3}{4} \text { if } \alpha=3
\end{array}\right.
$$

Let $x=\left(x_{1}, x_{2}\right)$ so that $x$ is a pure strategy for player I. Then,

$$
\begin{aligned}
M(G, x) \geqq & -\int_{\left(0, x_{1}\right]}\left(\frac{1}{2}-t_{2}\right) G\left(d t_{2}\right)-\int_{\left[1-x_{1}, 1\right)}\left(t_{2}-\frac{1}{2}\right) G\left(d t_{2}\right) \\
& -\int_{[0,1 / 2]}\left|\frac{1}{4}-t_{2}\right| G\left(d t_{2}\right)-\int_{[1 / 2,1]}\left|\frac{3}{4}-t_{2}\right| G\left(d t_{2}\right) \\
> & -\varepsilon-\frac{1}{4}
\end{aligned}
$$

so that

$$
\inf _{G} \sup _{F} M(F, G) \geqq-\frac{1}{4}
$$

and the game has no value.

In Ville's example the payoff function is such as to force each player to attempt to choose a point closer to 1 than does his opponent without actually choosing 1 . It is impossible for either player to guarantee he will achieve this with any preassigned positive probability no matter what pure strategy his opponent may use. In the example just presented a similar situation arises on the first two moves. In Ville's example the competition to choose a point close to the endpoint is. 
a direct competition over payoff. In the present example this competition is over the information player I will receive, which, of course, helps determine the payoff. If on his first move player I chooses a point closer to 0 (but not 0 ) than the choice of his opponent is to both 0 and 1 , then he will obtain more accurate information about the location of his opponent's choice than would be the case otherwise. Player II is prevented from choosing an endpoint since to do so would be to give his opponent perfect information.

4. A counter-example with continuous $\phi_{i}$. In this section a counter-example will be presented in which the functions $\phi_{i}$ are all continuous. In this example $n=4$. Again a comparison will be made with Ville's example.

Let

$$
\begin{aligned}
\phi_{1}\left(t_{1}\right) & \equiv 0 ; \\
\phi_{2}\left(t_{1}, t_{2}\right) & =t_{1}\left(1-t_{1}\right) t_{2} ; \\
\phi_{3}\left(t_{1}, t_{2}, t_{3}\right) & =\left\{\begin{array}{rr}
0 \text { if } \min \left(t_{1}, 1-t_{1}\right) \leqq t_{2} \leqq \max \left(t_{1}, 1-t_{1}\right) ; \\
t_{2}\left(1-t_{2}\right)\left(t_{1}-t_{2}\right)\left|t_{1}-\frac{1}{2}\right| \text { if } t_{2}<t_{1}<\frac{1}{2} \\
t_{2}\left(1-t_{2}\right)\left[t_{1}-\left(1-t_{2}\right)\right] \mid & \text { or } \frac{1}{2}<t_{1}-\frac{1}{2} \mid \text { if } \frac{1}{2} \leqq t_{1} ; \\
f\left(t_{1}, t_{2}, t_{3}, t_{4}\right) & =\left|t_{1}-t_{4}\right|-10\left|t_{2}-t_{3}\right| .
\end{array}\right.
\end{aligned}
$$

Assume $t_{2} \neq 0$ or 1 . Then, $\phi_{3}\left(t_{1}, t_{2}, t_{3}\right)>0$ for $\min \left(t_{2}, 1-t_{2}\right)<t_{1}<1 / 2$ while $\phi_{3}\left(t_{1}, t_{2}, t_{3}\right)<0$ for $1 / 2<t_{1}<\max \left(t_{2}, 1-t_{2}\right)$. On the other hand, $\phi_{3}\left(t_{1}, t_{2}, t_{3}\right)=0$ otherwise.

Let $F$ be any strategy for player I. Fix $\varepsilon>0$ and let $\delta \in(0, \varepsilon)$ be sufficiently small so that $F_{1}(0, \delta]+F_{1}[1-\delta, 1)<\varepsilon$. Let

$$
y_{2}(\alpha)=\left\{\begin{array}{l}
\frac{1}{2} \text { if } \alpha=0 ; \\
\frac{1}{4} \text { if } \alpha>0 \\
\frac{3}{4} \text { if } \alpha<0 ，
\end{array}\right.
$$

Let $G$ assign probability $1 / 2$ to each of the pure strategies $\left(\delta, y_{2}\right)$ and $\left(1-\delta, y_{2}\right)$. Then, 


$$
\begin{aligned}
& M(F, G) \leqq \int_{[0, \delta]}\left(\frac{1}{2}-t_{1}\right) F_{1}\left(d t_{1}\right)+\int_{[1-\delta, 1]}\left(t_{1}-\frac{1}{2}\right) F_{1}\left(d t_{1}\right) \\
&+\int_{(\delta, 1 / 2)}\left|t_{1}-\frac{1}{4}\right| F_{1}\left(d t_{1}\right)+\int_{(1 / 2,1-\delta)}\left|t_{1}-\frac{3}{4}\right| F_{1}\left(d t_{1}\right) \\
&-10\left[F_{1}\{0\}+F_{1}\{1\}\right]\left[\frac{1}{2} \int\left|\delta-t_{3}\right| F_{2,0}\left(d t_{3}\right)\right. \\
&\left.\quad+\frac{1}{2} \int\left|1-\delta-t_{3}\right| F_{2,0}\left(d t_{3}\right)\right] \\
&<\frac{1}{2}\left[F_{1}\{0\}+F_{1}\{1\}\right]+\frac{1}{2} \varepsilon+\frac{1}{4}\left[1-\varepsilon-F_{1}\{0\}-F_{1}\{1\}\right] \\
& \quad-5\left[F_{1}\{0\}+F_{1}\{1\}\right]\left[\left(\frac{1}{2}-\delta\right)+\left(1-\delta-\frac{1}{2}\right)\right] \\
& \quad=\frac{1}{4}+\frac{1}{4} \varepsilon-\left[F_{1}\{0\}+F_{1}\{1\}\right]\left[5(1-2 \delta)-\frac{1}{4}\right] \\
&<\frac{1}{4}+11 \varepsilon
\end{aligned}
$$

so that $\sup _{F} \inf _{G} M(F, G) \leqq 1 / 4$.

Let $G$ be any strategy for player II. Fix $\varepsilon>0$ and let $\delta \in(0, \varepsilon) \cap(0,1 / 2)$ be sufficiently small so that $G_{1,0}(0, \delta)+G_{1,0}(1-\delta, 1)<\varepsilon$. Let $x_{2}(\alpha)=$ $\alpha /[\delta(1-\delta)]$ and let $F$ assign probability $1 / 2$ to each of the pure strategies $\left(\delta, x_{2}\right)$ and $\left(1-\delta, x_{2}\right)$. When player I uses the strategy $F$ the value of the nonpositive term in $f$ will always be zero. Thus,

$$
\begin{aligned}
M(F, G) \geqq & {\left[1-G_{1,0}(0, \delta)-G_{1,0}(1-\delta, 1)\right] } \\
& \times\left[\frac{1}{2} \int\left|\delta-t_{4}\right| G_{2,0}\left(d t_{4}\right)+\frac{1}{2} \int\left|1-\delta-t_{4}\right| G_{2,0}\left(d t_{4}\right)\right] \\
> & \frac{1}{2}(1-\varepsilon)\left[\left(\frac{1}{2}-\delta\right)+\left(1-\delta-\frac{1}{2}\right)\right] \\
> & \frac{1}{2}-\frac{3}{2} \varepsilon
\end{aligned}
$$

so that $\inf _{G} \sup _{F} M(F, G) \geqq 1 / 2$ and the game has no value.

Here again the primary competition between the players is to make their first moves as close to the endpoints as possible without actually choosing the endpoints. If player I is successful in choosing a point $t_{1}$ at least as close to one of the endpoints as is player II's choice, then player II will have less information about $t_{1}$ than would be the case otherwise. Player I is prevented from choosing an endpoint by the fact 
that if he does so he will get no information about his opponent's first move so that he cannot guarantee that he can keep the negative term close to zero. Player II is prevented from choosing an endpoint by the fact that when he does so the function $\phi_{3}$ will take the value zero no matter what his opponent does so that he will have no information about player I's first move.

5. The case of information sets which are unions of generalized intervals. The case to be considered here is that in which each $\phi_{i}$ takes only a finite number of values and each is constant only on sets which are finite unions of $i$-dimensional generalized intervals. This is the only case considered in this paper in which $n$ remains arbitrary.

Let the values of $\phi_{i}$ be $1,2, \cdots, m_{i}$. Let $P_{j} \phi_{i}^{-1}(k)$ be the projection on the $j$ th coordinate of $\phi_{i}^{-1}(k)$ where $j=1,2, \cdots, i$. The interval $[0,1]$ can be subdivided into disjoint sets $B_{j 1}, B_{j 2}, \cdots, B_{j l_{j}}$ such that for each $B_{j l}$ there exist $i_{1}, i_{2}, \cdots, i_{r}$ and $k_{1}, k_{2}, \cdots, k_{u}$, all integers, such that $t \in B_{j l}$ if, and only if, $t \in P_{j} \phi_{i}^{-1}(k)$ whenever $i \in\left\{i_{1}, i_{2}, \cdots, i_{r}\right\}$ and $k \in\left\{k_{1}, k_{2}, \cdots, k_{u}\right\}$ while $t \notin P_{j} \phi_{i}^{-1}(k)$ otherwise. Suppose $j$ is even so that player II makes the $j$ th move. Let $y=\left(y_{1}, y_{2}, \cdots, y_{[n / 2]}\right)$ and $y^{\prime}=$ $\left(y_{1}^{\prime}, y_{2}^{\prime}, \cdots, y^{\prime[n / 2]}\right)$ be any strategies for player II such that $y_{i}=y_{i}^{\prime}$ for $i \neq j / 2$ and if $y_{j / 2}(k) \in B_{j l}$, then $y_{j / 2}^{\prime}(k) \in B_{j l}$. For any pure strategy $x$ for player I we have $t_{i}(x, y)=t_{i}\left(x, y^{\prime}\right)$ for $i=1,2, \cdots, j-1$ since for these values of $i$ player II's moves are unchanged. If $t_{j}(x, y) \in B_{j l}$, then $t_{j}\left(x, y^{\prime}\right) \in B_{j l}$. Hence,

$$
\phi_{j}\left(t_{1}(x, y), t_{2}(x, y), \cdots, t_{j}(x, y)\right)=\phi_{j}\left(t_{1}\left(x, y^{\prime}\right), t_{2}\left(x, y^{\prime}\right), \cdots, t_{j}\left(x, y^{\prime}\right)\right)
$$

so that $t_{j+1}(x, y)=t_{j+1}\left(x, y^{\prime}\right)$. Suppose that $t_{i}(x, y)=t_{i}\left(x, y^{\prime}\right)$ for $i=$ $j+1, j+2, \cdots, i_{0}$. Then, $\phi_{i_{0}}\left(t_{1}(x, y), t_{2}(x, y), \cdots, t_{i_{0}}(x, y)\right)=\phi_{i_{0}}\left(t_{1}\left(x, y^{\prime}\right)\right.$, $\left.t_{2}\left(x, y^{\prime}\right), \cdots, t_{i_{0}}\left(x, y^{\prime}\right)\right)$ so that $t_{i_{0}+1}(x, y)=t_{i_{0}+1}\left(x, y^{\prime}\right)$. Thus, $t_{i}(x, y)=$ $t_{i}\left(x, y^{\prime}\right)$ for all $i \neq j$.

For each $j=1,2, \cdots, n-1$ fix $\delta_{j}>0$ and select points $t_{j 1}, t_{j 2}, \cdots, t_{j v_{j}}$ such that for any $t_{j} \in B_{j l}$ there exists $t_{j v} \in B_{j l}$ such that for any $t_{1}, t_{2}, \cdots$, $t_{j-1}, t_{j+1}, \cdots, t_{n}$ we have

$$
\begin{aligned}
& \mid f\left(t_{1}, t_{2}, \cdots, t_{j-1}, t_{j}, t_{j+1}, \cdots, t_{n}\right) \\
& \quad-f\left(t_{1}, t_{2}, \cdots, t_{j-1}, t_{j v}, t_{j+1}, \cdots, t_{n}\right) \mid<\delta_{j} .
\end{aligned}
$$

Select the $t_{j v}$ in such a way that as $\delta_{j} \downarrow$ the set of all the $t_{j v}$ increases monotonically.

Let the game $\mathscr{G}\left(\delta_{1}, \delta_{2}, \cdots, \delta_{i}\right)=\left(X\left(\delta_{1}, \delta_{2}, \cdots, \delta_{i}\right), Y\left(\delta_{1}, \delta_{2}, \cdots, \delta_{i}\right)\right.$, $\left.M_{\delta_{1}, \delta_{2}}, \ldots, \delta_{i}\right)$ be our original game with the $j$ th move for $j=1,2, \cdots, i$ restricted to $t_{j 1}, t_{j 2}, \cdots, t_{j v_{j}}$. In $\mathscr{G}\left(\delta_{1}, \delta_{2}, \cdots, \delta_{n-1}\right)$ the player who makes the $(n-1)$ st move has only a finite number of strategies so that $\mathscr{G}\left(\delta_{1}, \delta_{2}, \cdots, \delta_{n-1}\right)$ has a value (see Wald [2]). 
Suppose $\mathscr{G}\left(\delta_{1}, \delta_{2}, \cdots, \delta_{i-1}, \delta_{i}\right)$ has a value for all $\delta_{i}>0$. It follows, by a proof similar to Ville's, that $\mathscr{G}\left(\delta_{1}, \delta_{2}, \cdots, \delta_{i-1}\right)$ has a value. Thus, by induction, $\mathscr{G}$ will also have a value.

Acknowledgment. The author wishes to express his gratitude to Professor David Blackwell for suggesting the problem treated here and for his continued interest in its solution.

\section{REFERENCES}

1. J. Ville, Sur la théorie générale des jeux où intervient l'habilité des joueurs, note in Traité du Calcul des Probabilites et de ses Applications, by E. Borel, Tome IV, Fascicule II, Applications aux jeux de hasard, (1938), 105-113.

2. A. Wald, Generalization of a theorem by von Neumann concerning zero sum two person games, Ann. Math. 46 (1945), 281-286.

Michigan State University 


\section{PACIFIC JOURNAL OF MATHEMATICS}

\section{EDITORS}

David GILbarg

Stanford University

Stanford, California

\section{F. H. BRowneLL}

University of Washington

Seattle 5, Washington

\section{A. L. Whiteman}

University of Southern California Los Angeles 7, California

\section{J. PAIGe}

University of California

Los Angeles 24, California

\section{ASSOCIATE EDITORS}
E. F. BECKENBACH
T. M. CHERRY
D. DERRY

\author{
E. HEWITT \\ A. HORN \\ L. NACHBIN
}

\author{
M. OHTSUKA \\ H. L. ROYDEN \\ M. M. SCHIFFER
}

E. SPANIER

E. G. STRAUS

F. WOLF

\section{SUPPORTING INSTITUTIONS}

\author{
UNIVERSITY OF BRITISH COLUMBIA \\ CALIFORNIA INSTITUTE OF TECHNOLOGY \\ UNIVERSITY OF CALIFORNIA \\ MONTANA STATE UNIVERSITY \\ UNIVERSITY OF NEVADA \\ NEW MEXICO STATE UNIVERSITY \\ OREGON STATE COLLEGE \\ UNIVERSITY OF OREGON \\ OSAKA UNIVERSITY \\ UNIVERSITY OF SOUTHERN CALIFORNIA
}

\author{
STANFORD UNIVERSITY \\ UNIVERSITY OF TOKYO \\ UNIVERSITY OF UTAH \\ WASHINGTON STATE COLLEGE \\ UNIVERSITY OF WASHINGTON \\ AMERICAN MATHEMATICAL SOCIETY \\ CALIFORNIA RESEARCH CORPORATION \\ HUGHES AIRCRAFT COMPANY \\ SPACE TECHNOLOGY LABORATORIES \\ NAVAL ORDNANCE TEST STATION
}

\footnotetext{
Mathematical papers intended for publication in the Pacific Journal of Mathematics should be typewritten (double spaced), and the author should keep a complete copy. Manuscripts may be sent to any one of the four editors. All other communications to the editors should be addressed to the managing editor, L. J. Paige at the University of California, Los Angeles 24, California.

50 reprints per author of each article are furnished free of charge; additional copies may be obtained at cost in multiples of 50 .
}

The Pacific Journal of Mathematics is published quarterly, in March, June, September, and December. The price per volume (4 numbers) is $\$ 12.00$; single issues, $\$ 3.50$. Back numbers are available. Special price to individual faculty members of supporting institutions and to individual members of the American Mathematical Society: $\$ 4.00$ per volume; single issues, $\$ 1.25$.

Subscriptions, orders for back numbers, and changes of address should be sent to Pacific Journal of Mathematics, 2120 Oxford Street, Berkeley 4, California.

Printed at Kokusai Bunken Insatsusha (International Academic Printing Co., Ltd.), No. 6, 2-chome, Fujimi-cho, Chiyoda-ku, Tokyo, Japan.

PUBLISHED BY PACIFIC JOURNAL OF MATHEMATICS, A NON-PROFIT CORPORATION

The Supporting Institutions listed above contribute to the cost of publication of this Journal, but they are not owners or publishers and have no responsibility for its content or policies. 


\section{Pacific Journal of Mathematics}

\section{Vol. 10, No. $4 \quad$ December, 1960}

M. Altman, An optimum cubically convergent iterative method of inverting a linear bounded operator in Hilbert space . . . . . . . . . . . . . . . . . . . . . . . . . . 1107

Nesmith Cornett Ankeny, Criterion for rth power residuacity ................. 1115

Julius Rubin Blum and David Lee Hanson, On invariant probability measures I . . . . . 1125

Frank Featherstone Bonsall, Positive operators compact in an auxiliary topology ..... 1131

Billy Joe Boyer, Summability of derived conjugate series . . . . . . . . . . . . . . . . 1139

Delmar L. Boyer, A note on a problem of Fuchs . . . . . . . . . . . . . . . . . 1147

Hans-Joachim Bremermann, The envelopes of holomorphy of tube domains in infinite

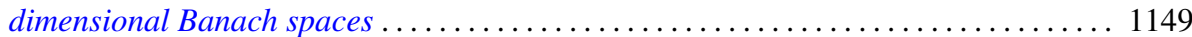

Andrew Michael Bruckner, Minimal superadditive extensions of superadditive

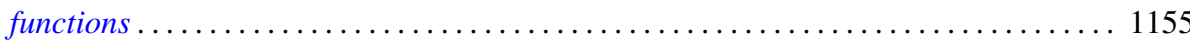

Billy Finney Bryant, On expansive homeomorphisms .................... 1163

Jean W. Butler, On complete and independent sets of operations in finite algebras . . . . . 1169

Lucien Le Cam, An approximation theorem for the Poisson binomial distribution ...... 1181

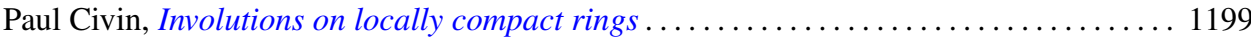

Earl A. Coddington, Normal extensions of formally normal operators . . . . . . . . . 1203

Jacob Feldman, Some classes of equivalent Gaussian processes on an interval ........ 1211

Shaul Foguel, Weak and strong convergence for Markov processes . . . . . . . . . . . 1221

Martin Fox, Some zero sum two-person games with moves in the unit interval ........ 1235

Robert Pertsch Gilbert, Singularities of three-dimensional harmonic functions . . . . . . . 1243

Branko Grünbaum, Partitions of mass-distributions and of convex bodies by

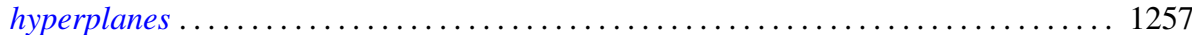

Sidney Morris Harmon, Regular covering surfaces of Riemann surfaces ........... 1263

Edwin Hewitt and Herbert S. Zuckerman, The multiplicative semigroup of integers

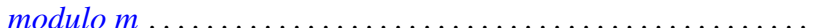

Paul Daniel Hill, Relation of a direct limit group to associated vector groups . ......... 1309

Calvin Virgil Holmes, Commutator groups of monomial groups . .

James Fredrik Jakobsen and W. R. Utz, The non-existence of expansive homeomorphisms

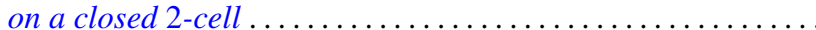

John William Jewett, Multiplication on classes of pseudo-analytic functions . . . . . . . 1323

Helmut Klingen, Analytic automorphisms of bounded symmetric complex domains . . . . 1327

Robert Jacob Koch, Ordered semigroups in partially ordered semigroups . . . . . . . . 1333

Marvin David Marcus and N. A. Khan, On a commutator result of Taussky and

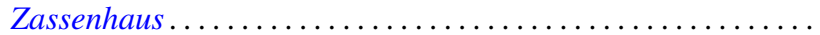

John Glen Marica and Steve Jerome Bryant, Unary algebras......

Edward Peter Merkes and W. T. Scott, On univalence of a continued fraction . . . . . . . 1361

Shu-Teh Chen Moy, Asymptotic properties of derivatives of stationary measures . . . . . 1371

John William Neuberger, Concerning boundary value problems . . . . . . . . . . . 1385

Edward C. Posner, Integral closure of differential rings . . . . . . . . . . . . . . . . . 1393

Marian Reichaw-Reichbach, Some theorems on mappings onto . . . . . . . . . . . . . 1397

Marvin Rosenblum and Harold Widom, Two extremal problems . . . . . . . . . . . . . . . . 1409

Morton Lincoln Slater and Herbert S. Wilf, A class of linear differential-difference

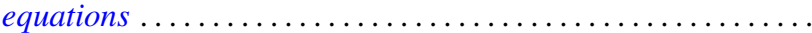

Charles Robson Storey, Jr., The structure of threads . . . . . . . . . . . . . . . . . . 1429

J. François Treves, An estimate for differential polynomials in $\partial / \partial z_{1},, \cdots, \partial / \partial z_{-} n \ldots \ldots 1447$

J. D. Weston, On the representation of operators by convolutions integrals . . . . . . . . 1453

James Victor Whittaker, Normal subgroups of some homeomorphism groups ......... 1469 